\title{
Does Red Cell Distribution Width Predict Outcome in Traumatic Brain Injury: Comparison to Corticosteroid Randomization After Significant Head Injury
}

\author{
Farid Sadaka ${ }^{\mathrm{a}, \mathrm{b}}$, Nicholas Doctors ${ }^{\mathrm{a}}$, Tallia Pearson ${ }^{\mathrm{a}}$, Brian Snyders ${ }^{\mathrm{a}}$, \\ Jacklyn O'Brien ${ }^{\mathrm{a}}$
}

\begin{abstract}
Background: Traumatic brain injury (TBI) is a leading cause of death and disability. The role of red cell distribution width (RDW) as a prognostic biomarker for outcome in TBI patients is unknown. Based on the corticosteroid randomization after significant head injury (CRASH) trial database, a prognosis calculator (CRASH) has been developed for outcome prediction in TBI. The objectives of this study are to investigate the association between RDW on day 1 of TBI and outcome, and to compare outcome prediction from RDW to that from CRASH.
\end{abstract}

Methods: We performed a retrospective review of patients with TBI and a Glasgow coma scale (GCS) score of 14 or less. Day 1 RDW and CRASH data were extracted. CRASH was calculated for each patient. Outcome was defined as mortality at 14 days and GOS at 6 months, with poor outcome defined as GOS of 1 - 3. Patients were stratified according to RDW values into six groups, and according to CRASH values into six groups.

Results: A total of 416 patients with TBI were included, with 339 survivors (S) and 77 non-survivors (NS). Compared to survivors, nonsurvivors were of similar age in years $(58 \pm 23$ vs. $58 \pm 23, \mathrm{P}=1.0)$, had lower GCS scores ( $5 \pm 3$ vs. $12 \pm 3, \mathrm{P}=0.0001)$, similar RDW $(14.0 \pm 1.2$ vs. $13.9 \pm 1.5, \mathrm{P}=0.6)$, and higher $\mathrm{CRASH}$ values $(68 \pm$ 26 vs. $24 \pm 22, \mathrm{P}=0.0001)$. Estimating the receiver-operating characteristic (ROC) area under the curve (AUC) showed that CRASH was a significantly better predictor of mortality compared to RDW (AUC $=0.91 \pm 0.01$ for CRASH compared to $0.66 \pm 0.03$ for RDW; $\mathrm{P}<0.0001)$. In addition, CRASH was a better predictor of neurologic outcome compared to RDW (AUC $=0.85 \pm 0.02$ for CRASH compared to $0.76 \pm 0.03$ for RDW; $\mathrm{P}=0.005$ ).

Conclusions: CRASH calculator was a strong predictor of mortality in patients with TBI. RDW on day 1 did not differ between survivors

Manuscript submitted September 14, 2017, accepted September 28, 2017

${ }^{a}$ Mercy Hospital St. Louis, St. Louis University, St. Louis, MO 63141, USA ${ }^{\mathrm{b} C o r r e s p o n d i n g ~ A u t h o r: ~ F a r i d ~ S a d a k a, ~ M e r c y ~ H o s p i t a l ~ S t . ~ L o u i s, ~ S t . ~ L o u i s ~}$ University, 625 S. New Ballas Rd, Suite 7020, St. Louis, MO 63141, USA. Email: farid.sadaka@mercy.net

doi: https://doi.org/10.14740/jocmr3173w and non-survivors, and was a poor predictor of mortality. Both RDW on day 1 and CRASH calculator are good predictors of 6-month outcome in TBI patients, although CRASH calculator remains a better predictor.

Keywords: Red cell distribution width; Traumatic brain injury; Mortality; Outcome

\section{Introduction}

Traumatic brain injury (TBI) is a major source of death and severe disability worldwide. In the USA alone, this type of injury causes 290,000 hospital admissions, 51,000 deaths, and 80,000 permanently disabled survivors [1-3].

Red blood cell distribution width (RDW) represents the size variation of all the red blood cells in an individual patient. RDW is calculated as the standard deviation in red blood cell (RBC) size divided by the mean corpuscular volume. RDW is ordered routinely as part of the complete blood count panel by an automated flow cytometry machine. RDW normally ranges between $11.5 \%$ and $14.5 \%$. Elevated RDW can result from any disease process that causes the premature release of reticulocytes into the circulation. Elevations in RDW have been shown to be associated with elevated inflammatory markers, such as C-reactive protein, erythrocyte sedimentation rate, and interleukin-6 [4,5]. Other studies showed that RDW predicted mortality in adults $>44$ years old in the general population [6], patients with cardiovascular disease, cancer, chronic lung diseases [7], symptomatic chronic congestive heart failure [8], acute stroke [9], acute heart failure [10], in the general critically ill patients [11], and in septic shock patients [12]. The role of RDW as a prognostic biomarker for neurologic outcome in TBI patients is unknown.

The corticosteroid randomization after significant head injury (CRASH) calculator has been validated to estimate mortality at 14 days and death and severe disability at 6 months (Glasgow outcome scale (GOS) 1 - 3). The calculator uses country of origin (USA in our dataset), age, Glasgow coma scale (GCS), pupils reactivity to light, presence of major extracranial injury, and findings on CT scan of brain (petechial hemorrhages, obliteration of the third ventricle or basal cis- 
Table 1. Comparison of Survivors to Non-Survivors

\begin{tabular}{llll}
\hline & Survivors & Non-survivors & P-value \\
\hline $\mathrm{N}, \%$ & $339(81.5)$ & $77(18.5)$ & \\
Age (mean \pm SD) & $58 \pm 23$ & $58 \pm 23$ & 1.0 \\
Gender (male, \%) & 63 & 69 & 0.3 \\
GCS (mean \pm SD) & $12 \pm 3$ & $5 \pm 3$ & 0.0001 \\
RDW (mean \pm SD) & $13.9 \pm 1.5$ & $14.0 \pm 1.2$ & 0.6 \\
CRASH (mean \pm SD) & $24 \pm 22$ & $68 \pm 26$ & 0.0001 \\
\hline
\end{tabular}

terns, subarachnoid bleeding, midline shift, and non-evacuated hematoma) [13]. The objectives of this study are to investigate the association between RDW on day 1 of TBI and outcome, and to compare outcome prediction from RDW to that from CRASH.

\section{Methods}

We performed a retrospective review of patients with TBI and a GCS score of 14 or less between January 2013 and September 2016. Day 1 RDW and CRASH data were extracted. CRASH was calculated for each patient. Patient identifiers were removed from the database, and informed consent was waived by our institutional review board. Mean, standard deviation and $\mathrm{P}$ values were reported for comparisons. Wilcoxon and Chi-squared statistics were used to determine significance. Significance was considered at the $\mathrm{P}<0.05$ level. The $\mathrm{C}$ statistic was calculated as a measure of the overall strength of prediction for both RDW and CRASH. Receiver-operating characteristic (ROC) curves were used to assess the mortality predictions from RDW on day 1 and CRASH. GOS was determined at 6 months. Neurologic outcome was scored according to the five-category GOS [14] for each patient. Patients were stratified according to RDW values into six groups, and according to CRASH values into six groups. ROC curves were used to assess GOS predictions from RDW on day 1 and

Predicting Mortality from RDW



1 - Specificity

\section{CRASH.}

\section{Results}

A total of 416 patients with TBI were included, with 339 survivors (S) and 77 non-survivors (NS); mortality rate was $18.5 \%$. Compared to survivors, non-survivors were of similar age in years $(58 \pm 23$ vs. $58 \pm 23, \mathrm{P}=1.0)$, similar gender (male, $69 \%$ vs. $63 \%, \mathrm{P}=0.3$ ), had lower GCS scores $(5 \pm 3$ vs. $12 \pm 3, \mathrm{P}=0.0001)$, similar RDW (14.0 \pm 1.2 vs. $13.9 \pm$ $1.5, \mathrm{P}=0.6)$, and higher CRASH values ( $68 \pm 26$ vs. $24 \pm 22$, $\mathrm{P}=0.0001$, higher values predict worse outcomes) (Table 1). Estimating the ROC area under the curve (AUC) showed that CRASH was a significantly better predictor of ICU mortality compared to RDW (AUC $=0.91 \pm 0.01$ for CRASH compared to $0.66 \pm 0.03$ for RDW; $\mathrm{P}<0.0001$ ) (Fig. 1). RDW was a good predictor of 6-month outcome (GOS 1 - 3), compared to $\mathrm{RDW}<13 \%$ group, $13-13.9 \%$ (odds ratio (OR): $8.0, \mathrm{P}=$ 0.005), 14-14.9\% (OR: 18, $\mathrm{P}<0.0001$ ), 15-15.9\% (OR: 10, $\mathrm{P}<0.001), 16-16.9 \%$ (OR: $4.3, \mathrm{P}=0.03)$, and $>17 \%(\mathrm{OR}$ : $6.7, \mathrm{P}<0.01)$. CRASH was also a strong predictor of 6 -month outcome (GOS 1 - 3), compared to CRASH $<10$ group, 11 30 (OR: 2, $\mathrm{P}=0.1), 31$ - 50 (OR: 6, $\mathrm{P}=0.01)$, 51 - 70 (OR: $16, \mathrm{P}=0.0001), 71-90$ (OR: 41, $\mathrm{P}<0.0001)$, and $>90$ (OR: $77, \mathrm{P}<0.0001$ ) (Table 2). Estimating the ROC AUC showed that CRASH was a better predictor compared to RDW (AUC $=0.85 \pm 0.02$ for CRASH compared to $0.76 \pm 0.03$ for RDW; $\mathrm{P}=0.005)$ (Fig. 2).

\section{Discussion}

Elevations in RDW have been shown to be associated with elevated inflammatory markers, for which reason it has been studied as a possible easily obtained predictor of outcome in several disease states. RDW predicted mortality in the general population [6], patients with cardiovascular disease, cancer, chronic lung diseases [7], symptomatic chronic congestive

Predicting Mortality from CRASH

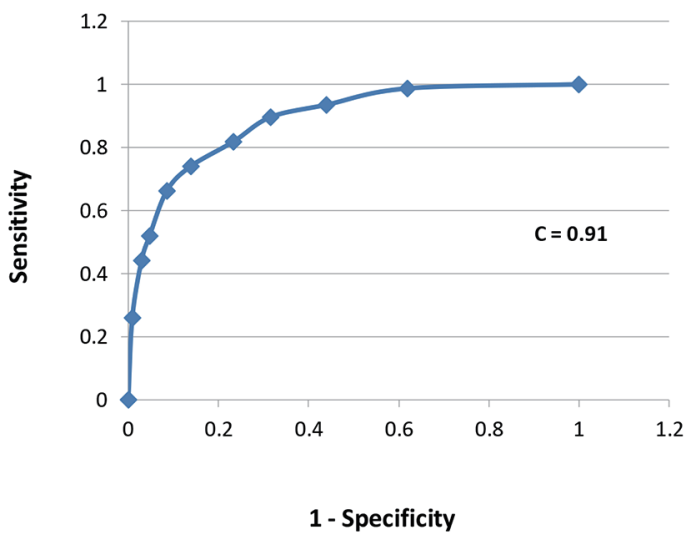

Figure 1. Receiver-operating characteristic curves for each of RDW and CRASH for prediction of mortality. 
Table 2. Association of RDW and CRASH With Poor Neurologic Outcome (GOS 1 - 3)

\begin{tabular}{|lll|}
\hline & Odds ratio & P value \\
\hline $\begin{array}{c}\text { Red cell distribution width }(\%) \\
<13\end{array}$ & 1.0 & \\
\hline $13-13.9$ & 8.0 & Reference \\
$14-14.9$ & 18 & 0.005 \\
$15-15.9$ & 10 & 0.0001 \\
$16-16.9$ & 4.3 & 0.001 \\
$>17$ & 6.7 & 0.03 \\
CRASH (\%) & & 0.01 \\
$<10$ & 1.0 & \\
$11-30$ & 2.0 & Reference \\
$31-50$ & 6.0 & 0.1 \\
$51-70$ & 16 & 0.01 \\
$71-90$ & 41 & 0.0001 \\
$>90$ & 77 & 0.0001 \\
\hline
\end{tabular}

heart failure [8], acute stroke [9], acute heart failure [10], the general critically ill patients [11], and in septic shock patients [12]. As a result, we elected to study its predictability of outcome in TBI patients. Only one previous study by Zhang et al looked at RDW's prediction of mortality in patients with TBI. In a retrospective analysis of 122 TBI patients, RDW was predictive of 28-day mortality, with a cut-off level for RDW $\geq 12.85$, and an AUC of 0.805 . Neurologic outcome was not evaluated [15]. In our study, RDW was not predictive of mortality, but was predictive of 6-month neurologic outcome. The reasons why our study differed from Zhang et al in mortality prediction could be a different patient population, variables not accounted for could be associated with inflammation and thus RDW, such as septic shock, diabetes, heart failure, cancer, chronic lung disease, etc. In addition,



our study was larger (416 vs. 122 patients) with less tendency for type II error. However, RDW in our study was predictive of neurologic outcome at 6 months. It is well known that the neuroinflammatory response following TBI is a key secondary injury factor that can drive ongoing neuronal injury and worsen outcome. Inflammation is associated with an increase in blood-brain barrier permeability and the development of vasogenic edema as well as neuronal injury and worse functional outcome $[16,17]$. As a result, RDW, being a marker of inflammation, could play a role in prediction of neurologic outcome in TBI patients.

Our study has several limitations. It is a retrospective study with a relatively small number of patients. In addition, a number of variables that could potentially influence outcome were not collected and accounted for (e.g. diabetes, sepsis, infections, heart failure, iron deficiency anemia, etc.). Although CRASH calculator has been validated in a large number of TBI patients, the degree of agreement likely varies substantially by population; therefore point estimates of agreement in terms of generalizability of our findings must be interpreted with caution especially because it is a retrospective single center study. Our study was performed in an academic referral hospital; therefore our results may not be applicable to institutions with different patient populations. The strengths of our study include the inclusion of 6 months neurologic outcome (only study of RDW prediction of neurologic outcome), and the comparison to a well-validated outcome prediction calculator in TBI (CRASH).

\section{Conclusion}

CRASH calculator was a strong predictor of mortality in patients with TBI. RDW on day 1 did not differ between survivors and non-survivors, and was a poor predictor of mortality. Both RDW on day 1 and CRASH calculator were good predictors of 6-month outcome in TBI patients, although CRASH calculator remains a better predictor. Pending larger studies to validate our findings, we believe that outcome predictors can



Figure 2. Receiver-operating characteristic curves for each of RDW and CRASH for prediction of neurologic outcome (GOS 1 - 3 at 6 months). 
only support - not replace - clinical judgment.

\section{Sponsorship}

This study was not sponsored.

\section{Disclosures}

None of the authors have anything to disclose.

\section{References}

1. Ghajar J. Traumatic brain injury. Lancet. 2000;356(9233):923-929.

2. Rutland-Brown W, Langlois JA, Thomas KE, Xi YL. Incidence of traumatic brain injury in the United States, 2003. J Head Trauma Rehabil. 2006;21(6):544-548.

3. Kermer P, Klocker N, Bahr M. Neuronal death after brain injury. Models, mechanisms, and therapeutic strategies in vivo. Cell Tissue Res. 1999;298(3):383-395.

4. Lippi G, Targher G, Montagnana M, Salvagno GL, Zoppini G, Guidi GC. Relation between red blood cell distribution width and inflammatory biomarkers in a large cohort of unselected outpatients. Arch Pathol Lab Med. 2009;133(4):628-632.

5. Forhecz Z, Gombos T, Borgulya G, Pozsonyi Z, Prohaszka Z, Janoskuti L. Red cell distribution width in heart failure: prediction of clinical events and relationship with markers of ineffective erythropoiesis, inflammation, renal function, and nutritional state. Am Heart J. 2009;158(4):659-666.

6. Patel KV, Ferrucci L, Ershler WB, Longo DL, Guralnik JM. Red blood cell distribution width and the risk of death in middle-aged and older adults. Arch Intern Med. 2009;169(5):515-523.

7. Perlstein TS, Weuve J, Pfeffer MA, Beckman JA. Red blood cell distribution width and mortality risk in a community-based prospective cohort. Arch Intern Med. 2009;169(6):588-594.

8. Felker GM, Allen LA, Pocock SJ, Shaw LK, McMurray JJ, Pfeffer MA, Swedberg K, et al. Red cell distribution width as a novel prognostic marker in heart failure: data from the CHARM Program and the Duke Databank. J Am Coll Cardiol. 2007;50(1):40-47.

9. Ani C, Ovbiagele B. Elevated red blood cell distribution width predicts mortality in persons with known stroke. J Neurol Sci. 2009;277(1-2):103-108.

10. van Kimmenade RR, Mohammed AA, Uthamalingam S, van der Meer P, Felker GM, Januzzi JL, Jr. Red blood cell distribution width and 1-year mortality in acute heart failure. Eur J Heart Fail. 2010;12(2):129-136.

11. Bazick HS, Chang D, Mahadevappa K, Gibbons FK, Christopher KB. Red cell distribution width and allcause mortality in critically ill patients. Crit Care Med. 2011;39(8):1913-1921.

12. Sadaka F, O'Brien J, Prakash S. Red cell distribution width and outcome in patients with septic shock. J Intensive Care Med. 2013;28(5):307-313.

13. Perel P, Arango M, Clayton T, Edwards P, Komolafe E, Poccock S, Roberts I, et al. Predicting outcome after traumatic brain injury: practical prognostic models based on large cohort of international patients. BMJ. 2008;336(7641):425-429.

14. Jennett B, Bond M. Assessment of outcome after severe brain damage. Lancet. 1975;1(7905):480-484.

15. Zhang B, Zhao J. Red blood cell distribution width as a prognostic biomarker for mortality in traumatic brain injury. Int J Clin Exp Med. 2015;8(10):19172-19175.

16. Corrigan F, Mander KA, Leonard AV, Vink R. Neurogenic inflammation after traumatic brain injury and its potentiation of classical inflammation. J Neuroinflammation. 2016;13(1):264.

17. Woodcock T, Morganti-Kossmann MC. The role of markers of inflammation in traumatic brain injury. Front Neurol. 2013;4:18. 